\title{
A COMPARATIVE STUDY \\ OF THREE COLLOIDAL REACTIONS ON THE CEREBROSPINAL FLUID.
}

\section{- By D. O. RIDDEL and R. M. STEWART, Whttixgham.}

THE colloidal-gold reaction introduced by Lange in 1912 is now generally accepted as one of the most important laboratory procedures in the examination of the cercbrospinal fluid. In neuro-syphilis it gives results which rival in value those of the Wasscrmann reaction, and its employment as a diagnostic aid in many non-syphilitic diseases has a wide sphere of utility which even at the present day does not appear to be fully appreciated.

Although the difficulties which at first surrounded the preparation of the reagent have now been largely overcome, there is still an element of uncertainty in its manufacture which cannot be altogether explained by failure to obtain a perfectly neutral solution. It occasionally happens that an apparently good reagent, fulfilling all the standard requirements laid down by Miller and his associates, ${ }^{1}$ behaves in an irregular and mystifying manner, and, when such a misfortune arises, the worker must perforce reject his results and repeat from the beginning the rather laborious preparation of a new stock of solution. With a view to eliminating the risk of failure which attends the preparation of a solution of colloidal gold, proposals have been made to substitute other colloidal solutions which do not require the absolute purity of reagents and glassware which is so essential in Lange's test. It is perhaps too early to place much reliance on the trustworthiness of these modifications; but, judging from the few papers which have appeared, there seems to be a definite consensus of opinion that the colloidal tests in which gum mastic and benzoin are employed show a degree of parallelism to the gold test sufficiently close to justify their more extended use. One of the difficulties met with in attempting to apprize the value of these new tests is that few workers have carried out comparative tests, and in this country only Lange's reaction appears to have attracted attention. We have therefore thought it desirable to present the results of a short comparative study of the gold, the gum-mastic, and the colloidal-benzoin reactions, together with certain observations which may prove of interest to those performing these tests. 
Material.- The comparative curves presented in this paper are 100 in number. The spinal fluids were obtained from asylum inmates, and, since the reactions with which we are here concerned find their greatest application in neuro-syphilis, a relatively large percentage of general paralytics have been included. None of the patients had received antisyphilitic treatment prior to examination of their fluids.

Technique.--

The Colloidal-Gold Reagent was prepared according to the standard method of Miller, Brush, Hammers, and Felton. Each fresh batch of reagent was tested with the spinal fluid of a known general paralytic, and the test was performed in the usual manner. All fluids were examined within a few hours of their withdrawal.

The Colloidal-Mastic Test of Emanuel was employed in the manner modified by Cutting, ${ }^{2}$ using Smith's ${ }^{3}$ numerical scale to denote degrees of flocculation and precipitation. The emulsion was prepared from the stock solution (10 grm. gum mastic in 100 c.c. absolute alcohol) by diluting 1 c.c. of the latter with 9 c.c. of absolute alcohol; 40 c.c. of distilled water were placed in a small flask, and the diluted mastic solution rapidly added and mixed by rotation. To the first of eleven small test tubes ( $3 \frac{1}{2}$ in. $\times \underset{2}{1}$ in.) there was added 1.5 c.c. of a salt solution containing 99 c.c. of $\mathbf{1} \cdot 25$ per cent sodium chloride plus 1 c.c. of 0.5 per cent potassium carbonate, and to each of the remainder 1 c.c. of the same solution; 0.5 c.c. of cerebrospinal fluid was next added to the first tube, and, after mixing, 1 c.c. transferred to the second tube, and so on to the tenth tube; from this 1 c.c. was rejected, the eleventh tube thus serving as a control. Finally, to each of the eleven tubes 1 c.c. of the mastic solution was added. The tubes were allowed to stand for twelve hours at room temperature and the results then read.

The Colloidal-Benzoin Test of Guillain, Laroche, and Lechelle ${ }^{4}$ is carried out as follows: Two stock solutions are prepared, one containing 0.01 per cent chemically pure sodium chloride, and the other a suspension of benzoin resin. One gramme of benzoin resin is dissolved in 10 c.c. of absolute alcohol, and after the lapse of forty-eight hours the supernatant fluid is decanted; of this, $0 \cdot 3$ c.c. is added drop by drop to 20 c.c. of distilled water, and the emulsion then heated to $35^{\circ} \mathrm{C}$. in order to obtain a homogeneous suspension. The dilutions are carried out as follows: Sixteen small test tubes are set up in a rack; in the first tube there is placed 0.25 c.c. of the saline solution, in the second 0.5 c.c., and in the third 1.5 c.c.; each of the remaining tubes receives 1 c.c. of the same solution. Cerebrospinal fluid is next added : 0.75 c.c. to the first tube, 0.5 c.c. to the second and third tubes. From the latter, after mixing, 1 c.c. is transferred to the fourth tube, and so on until the fifteenth tube is reached. The cubic centi- 
metre from it is rejected, the sixteenth tube thus acting as a control. Lastly, to each of the sixteen tubes 1 c.c. of benzoin solution is added. The dilutions obtained are thus in geometrical progression, and range from 1-2 in the second tube to 1-16,384 in the fifteenth tube.

Graphic Representation.-

Gold-Sol Test.--In recording results the numeral 5 is used to indicate complete precipitation, and the lesser values to designate the graduated colour changes in the tubes where partial precipitation has occurred. In positive reactions precipitation always begins in tubes 3 and 4, and the tubes must be allowed to stand for twelve hours. before the results are read.

A negative reaction is shown by an absence of colour change in all the tubes. The so-called paretic curve shows complete precipitation in the first few tubes, giving a colourless solution, with lesser changes in the remaining tubes. A typical paretic curve gives the

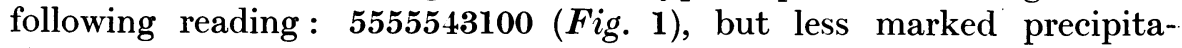
tion may also be accepted where the general conformation of the curve-

Lange Gold-Sol Test.

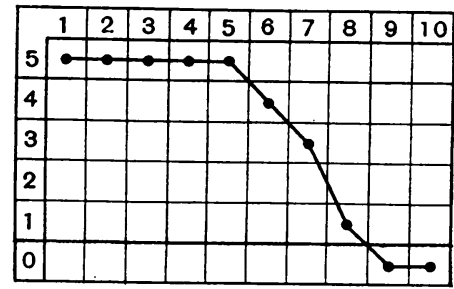

Fig. 1.-Paretic curve.

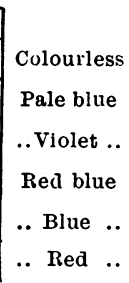

.. 0

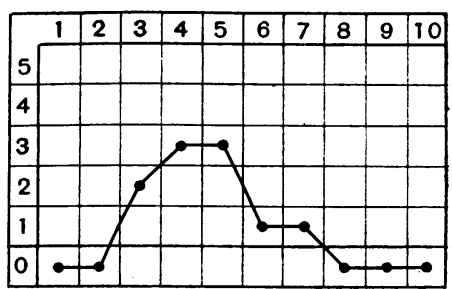

FIG. 2.-Syphilitic curve.

is preserved; thus, a reading $\mathbf{4 4 4 4 4 3 2 1 0}$ is still fairly typical. In general it may be said that where reduction in the first three or more tubes is well marked it may be considered a paretic curve. The syphilitic curve shows a complete absence of precipitation in the first one or two tubes, with the maximum colour change (usually violet and seldom beyond pale blue) in the fourth or fifth tube. A typical curve would be 0023311000 (Fig. 2), and usually tabes dorsalis and cerebrospinal syphilis conform to this type.

Gum-Mastic Test.-The maximum change in this test is indicated by 4 (Smith's scale), and the lesser numerical values differ proportionately. A negative result is indicated by a total absence of precipitation in all the tubes, and a paretic curve is shown by complete precipitation in the first three or more tubes : $\mathbf{4 4 4 4 4 3 1 0 0 0}$ (Fig. 3) ; but lesser degrees of precipitation may still be accepted provided the general conformation is preserved.

With regard to the syphilitic curve, the gum-mastic test does not appear to give any constant reading, and in this respect is much voL. II.-No. 8. 
inferior to the gold-sol test. In meningo-vascular syphilis, partial precipitation usually occurs in the first three or four tubes, but the first tube not infrequently remains unaltered. A curve such as 1332000000 (Fig. 4) might be interpreted as syphilitic or tabetic, but the wide variations encountered do not permit of a definite ruling on this point.

Colloidal-Mastic Test.

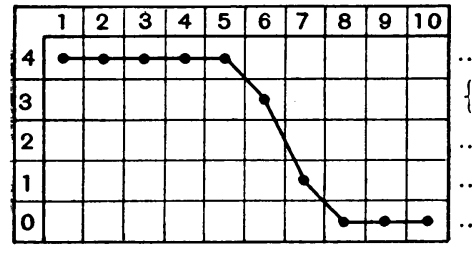

FIG. 3.-Paretic curve.

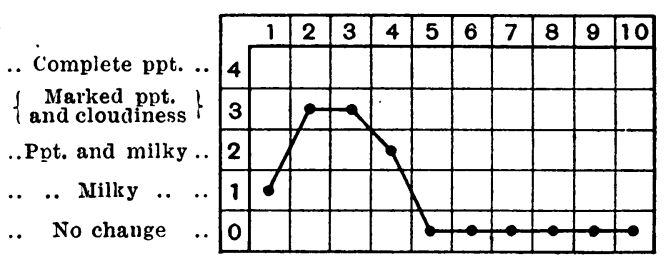

FIG. 4.-Syphilitic curve.

The Colloidal-Benzoin Test.-A negative reaction is indicated on a vertical line by the figure $0 ; 1$ indicates a weak positive where the fluid remains cloudy but shows precipitation; 2 indicates a positive reaction where precipitation is complete. The tubes are allowed to remain at room temperature for six hours before the results are read. As in the other tests, a negative result is shown by an absence of change in the fifteen tubes.

Colloidal-Benzoin Test.

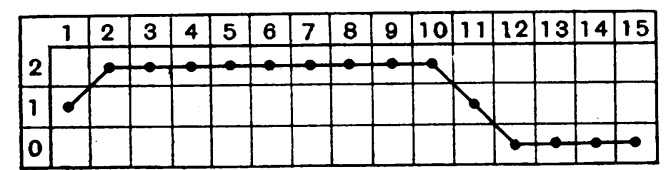

FIG. 5.-Paretic curve.

\begin{tabular}{|c|c|c|c|c|c|c|c|c|c|c|c|c|c|}
\hline & & 2 & 3 & 4 & 5 & 6 & 7 & 8 & 9 & \begin{tabular}{|l|l|}
10 & 11 \\
\end{tabular} & 12 & 13 & \begin{tabular}{l|l}
14 & 15 \\
\end{tabular} \\
\hline 2 & & & & & & & & & & & & & \\
\hline 1 & & & & 7 & & & & & & & & & \\
\hline 10 & & & & & $\theta$ & & $\rightarrow$ & & & 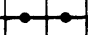 & & -1 & • \\
\hline
\end{tabular}

FIG. 6.-Syphilitic curve.

In their description of this test the authors recorded their results as positive or negative, no tables being provided to show the degrees of precipitation in positive reactions. They state that a paretic benzoin curve shows precipitation in the first nine or occasionally thirteen tubes. Apparently it is not essential that reduction should be absolute in all nine tubes, and the number showing complete precipitation may be increased or decreased somewhat without altering the interpretation. Duhot and Crampon ${ }^{5}$ regard as positive a reaction in which precipitation occurs in the first four tubes. In our series 
we have accepted as paretic a curve in which precipitation is complete or almost complete in five out of the first six tubes (Fig. 5).

A syphilitic curve is indicated by a partial precipitation in tubes 1 to 3 or 4 (Fig. 6).

Limiting Factors in the Three Tests.-In all three tests cerebrospinal fluid which has been standing for some time will give curves differing from those made with fresh fluid. This effect is least noticeable with the colloidal-gold solution, and in this test fluids kept at room temperature may be used up to forty-eight hours after withdrawal. Blood-contaminated fluids introduce serious errors in interpretation and should not be employed. In the case of Lange's test, spinal fluid containing blood in macroscopic quantities may give reactions in the luetic zone, and in the other two tests blood-admixture gives rise to irregular curves. The colloidal-gold reaction may be performed with old solutions, provided they have been kept well corked in a dark cupboard. The colloidal solutions of mastic and benzoin require to be freshly prepared; those older than a few days give unsatisfactory results. It may also be mentioned here that not every sample of gum mastic appears suitable for the test, but we have found samples of ordinary commercial benzoin resin quite satisfactory.

Results of Authors' Tests.-In the following analysis we have grouped the cases for purposes of study under the following divisions : (1) Dementia paralytica ; (2) Tabes dorsalis; (3) Cerebrospinal syphilis; (4) Disseminated sclerosis; (5) Non-syphilitic disease of the nervous system ; (6) Miscellaneous.

Wassermann tests were performed only where the clinical diagnosis was in doubt or in cases in which the colloidal tests suggested an unsuspected syphilitic involvement of the nervous system.* For determining the presence or absence of globulin in the spinal fluid, reliance was placed on the Ross-Jones and Pandy tests. For the cell count the method described by Bybee and Lorenz ${ }^{6}$ was employed.

1. General Paralysis (Table I).- The spinal fluids of 30 cases of general paralysis were examined, and all three tests yielded typical curves in 28 instances. In the remaining 2 cases, which presented well-marked physical signs, the gold and benzoin tests were positive, and the mastic negative or atypical. The complete uniformity of the gold and benzoin reactions was very striking, and suggests that they are probably of equal value in the diagnosis of dementia paralytica. On the other hand, a paretic gold curve was obtained in four fluids taken from patients presenting no symptoms of general paralysis.

* The Wassermann reactions were performed at the City Laboratories, Liverpool, under the direction of Professor J. M. Beattie. 
The first case was a demented female, 70 years old, who had suffered from a peripheral neuritis affecting the legs in 1916. Her blood and spinal fluid Wassermann were both negative. The second patient, although diagnosed general paralysis on admission, and in several other institutions, was unquestionably a case of disseminated sclerosis. He gave a negative Wassermann reaction in both blood and cerebrospinal fluid. The remaining fluids were from two cases of tabes, and in both the Wassermann reaction was strongly positive in the cerebrospinal fluid. The mental state of the first had been one of delusional insanity for nine years, and there was nothing to suggest an associated syphilitic involvement of the brain. The second case was one of progressive tabes with melancholia.

The gum-mastic reaction gave paretic curves in two non-syphilitic fluids-the chronic demented patient, and the case of disseminated sclerosis. The colloidal-benzoin reaction gave no paretic curves in non-syphilitic cases. From this brief analysis it is seen that in no instance were there paretic mastic and benzoin curves with a negative gold curve, and that, although the gold and mastic tests gave paretic curves in disseminated sclerosis, the benzoin reaction failed to do so.

It is not a little remarkable that in one instance two reactions gave paretic curves in the absence of other positive laboratory findings or clinical manifestations of syphilis. The patient was a woman, age 71 , in an advanced stage of dementia; alcohol was the supposed cause of her insanity, and in 1916 she developed peripheral neuritis affecting the legs, which left them permanently paralyzed in a state of talipes equinus.

Tabes Dorsalis (Table 2).- - The 3 cases that were diagnosed tabes dorsalis presented well-marked signs and symptoms of the disease. The mastic and benzoin tests gave syphilitic curves in the three fluids, but the colloidal-gold reaction gave in two instances paretic curves to which reference has already been made; in the other fluid a wellmarked syphilitic curve was obtained.

Cerebrospinal Syphilis (Table 3).-The colloidal-gold reaction gave curves in the syphilitic zone in all 6 cases; the mastic test also gave curves which were interpreted as syphilitic. The colloidalbenzoin test showed syphilitic curves in 4 cases and negative in two fluids. The Wassermann reaction proved positive in 5 cases and negative in the sixth. We have included the latter case in the cerebrospinal group because the clinical history and physical state of the patient pointed clearly to a syphilitic infection of the nervous system. He contracted syphilis when 22 years of age, and had a transient attack of hemiplegia four years later. A mild dementia supervened, and he is now in his thirty-seventh year. Although both the Wassermann and 
benzoin reactions are negative, it is probable that the positive gold curve is the more correct index of his condition.

Syphilitic Curves in Non-syphilitic Disease (Table 3a).-1. A primary dement with symmetrical wasting of the hands and slight spastic paresis of the lower limbs gave a syphilitic type of curve with the colloidal-gold solution, and negative results in the mastic and benzoin tests (Wassermann -, cells 4 , globulin faintly + ).

2. A patient with chronic tuberculous adenitis and recent symmetrical swelling of the parotid glands gave negative gold and benzoin curves and a positive mastic curve (Wassermann -, cells 6, globulin faintly + ).

3. One case of insanity with epilepsy gave a syphilitic gold curve, slight reduction in the mastic test, and a negative colloidal benzoin reaction (Wassermann -, cells o, globulin -).

4. In a case of confusional insanity the colloidal-gold reaction showed reduction in the syphilitic zone, a mastic curve which was interpreted as syphilitic, and a negative colloidal-benzoin reaction (Wassermann -, cells 8, globulin -).

The above four cases form too small a group on which to base any conclusions, but the parallelism between the Wassermann reaction and benzoin test is certainly noteworthy.

Disseminated Sclerosis (Table 4).--..The first case gave syphilitic curves in all three reactions. The spinal fluid of the second case, to whom reference has already been made, showed a paretic curve in the gold and mastic tests, and a syphilitic curve in the benzoin test.

Organic Nerious Diseases (Table 5). -With two exceptions all three tests were negative. A patient suffering from pseudobulbar paralysis showed very slight reduction in the middle zone in the colloidal-gold test, slight reduction in the first two tubes of the mastic test, and a negative colloidal-benzoin reaction (Wassermann -, cells 4, globulin faintly + ). In another patient suffering from paraplegia the gold test was negative, the mastic showed slight reduction in the first five tubes, and the benzoin test was negative (Wassermann -, cells 10, globulin faintly + ).

Miscellaneous (Table 6).-A survey of this group shows that no positive results were obtained.

Relation of the Globulin Content and Cell Count to the Thiee Reactions.-In 30 cases of dementia paralytica, positive colloidal reactions were in all instances associated with pleocytosis and increased globulin content; but on the other hand, in 12 cases in which all three tests were negative there was a definite increase in globulin, and in 13 cases giving negative results the cell-count was increased. One fluid with no increase of globulin gave paretic curves in the colloidal-gold and gum-mastic tests, and in one case of dementia in whom all three tests 
were positive the globuijn content was within normal limits. In only one instance did we find all three reactions negative in a spinal fluid which showed a positive Wassermann reaction, pleocytosis, and increase of globulin. In other words, the three colloidal reactions failed to give evidence of a syphilitic infection in 1 per cent of the series. The patient was a woman with gummatous ulceration of the soft palate, and curiously enough her blood Wassermann was negative.

Table 1._General Paralysis.

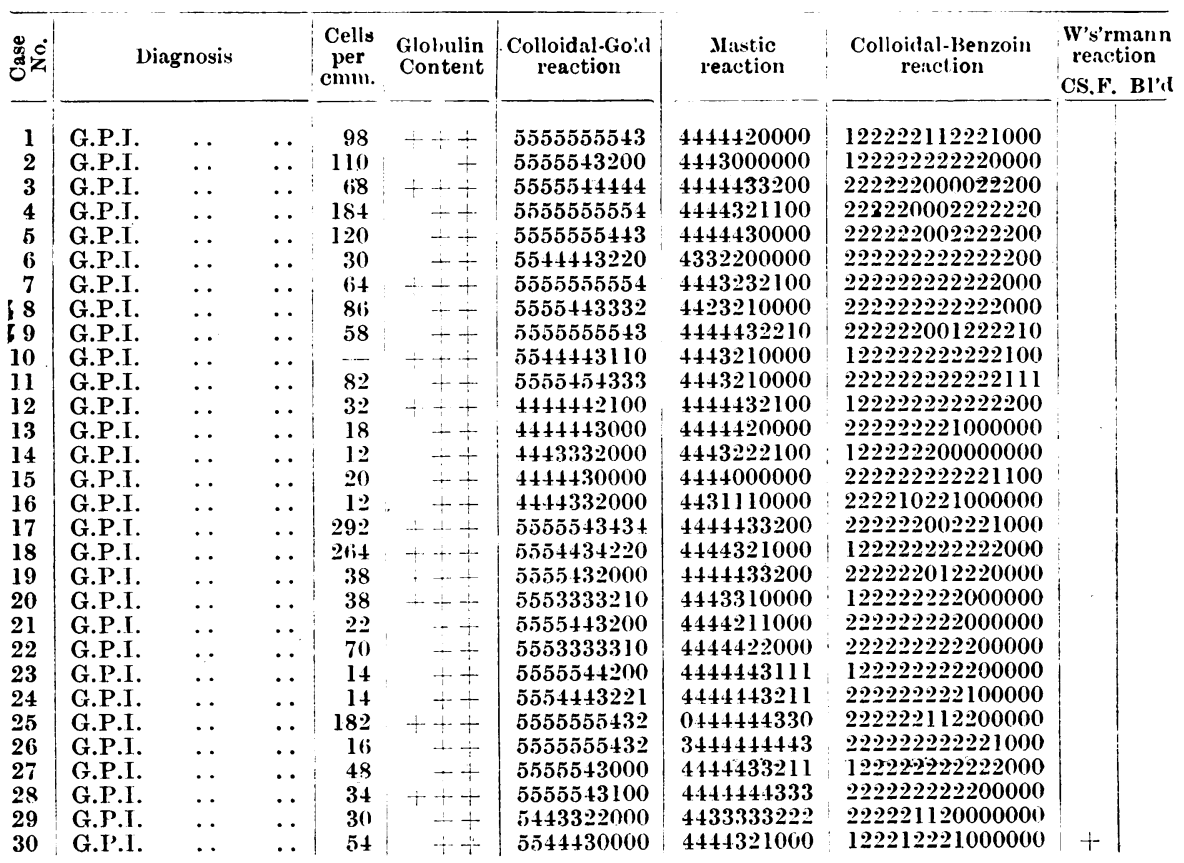

Table 2.-Tabes Dorsalis.

$31 \mid$ Tabes dorsalis $\ldots . \quad 60$

Tabes dorsalis

33 Tabes dorsalis

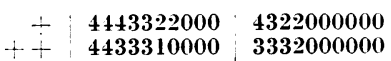

$-\quad 1133311000$ 2332100000

Table 3.-Cerebrospinal Syphilis.

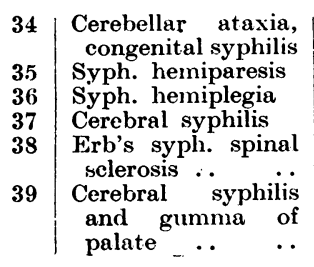

\begin{tabular}{|c|c|}
\hline 34 & $\begin{array}{l}\text { Cerebellar ataxia, } \\
\text { congenital syphilis }\end{array}$ \\
\hline 35 & Syph. hemiparesis \\
\hline 36 & Syph. hemiplegia \\
\hline 37 & Cerebral syphilis \\
\hline 38 & $\begin{array}{l}\text { Erb's syph. spinal } \\
\text { sclerosis .. }\end{array}$ \\
\hline 39 & $\begin{array}{c}\text { Cerebral syphilis } \\
\text { and gumma of } \\
\text { palate } \ldots\end{array}$ \\
\hline
\end{tabular}

012200222100000

111102222100000

111111220000000

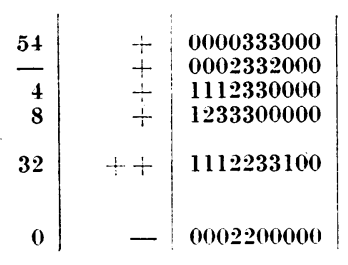

3322000000
3210000000
3332221100
4322000000
2344432100
3322100000




Table 3a.-Syphilitic Curves in Non-syphilitic Disease.

\begin{tabular}{|c|c|c|c|c|c|c|c|c|}
\hline 总安 & Diagr.osis & $\begin{array}{c}\text { Ceils } \\
\text { per } \\
\text { cnim. }\end{array}$ & $\begin{array}{l}\text { Globulin } \\
\text { Content }\end{array}$ & $\begin{array}{l}\text { Colloidal-Gold } \\
\text { reaction }\end{array}$ & $\begin{array}{l}\text { Mastic } \\
\text { reaction }\end{array}$ & $\begin{array}{c}\text { Colloidal-Benzoin } \\
\text { reaction }\end{array}$ & $\begin{array}{l}\text { W's'rn } \\
\text { react } \\
\text { CS.F. }\end{array}$ & $\begin{array}{l}\operatorname{mann} \\
\text { tion } \\
\text { Bl'd }\end{array}$ \\
\hline 40 & Muscular atrophy & & & & & & & \\
\hline 41 & $\begin{array}{l}\text {-hands } \ddot{\text { with epi- }} \text {. } \\
\text { Insanity }\end{array}$ & 4 & $\therefore$ & 0022210000 & 0000000000 & 000001222000000 & - & $\vdots$ \\
\hline $\begin{array}{l}42 \\
43\end{array}$ & lepsy & 0 & - & $\begin{array}{l}1122210000 \\
1123000000\end{array}$ & $\begin{array}{l}3300000000 \\
4421002000\end{array}$ & 000000000000000 & - & \\
\hline
\end{tabular}

Table 4.-Disseminated Sclerosis.

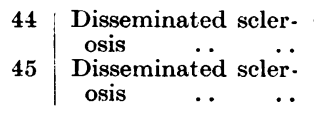

0

$+\quad$\begin{tabular}{l|l|}
5444321000 & 1443210000 \\
-1003444100 & 3320000000
\end{tabular}

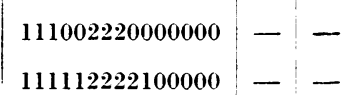

Table 5.-Organic Nervous Diseases.

\begin{tabular}{|c|c|}
\hline 46 & Motor aphasia \\
\hline 17 & Pseudobulbar para- \\
\hline 48 & $\begin{array}{l}\text { lysis } \\
\text { Infantile }\end{array}$ \\
\hline & paralysis \\
\hline 49 & Spastic paralysis.. \\
\hline () & Hemiplegia \\
\hline & Hemiplegia - epi- \\
\hline & leptic imbecile .. \\
\hline 2 & Hemiplegia \\
\hline & Paraplegia \\
\hline 4 & Triplegia \\
\hline & stenosis) . . \\
\hline & Hemiplegia \\
\hline 6 & Hemiplegia \\
\hline & Infantile \\
\hline & myelitis $\cdots$ \\
\hline 58 & Facial hemispasm \\
\hline 9 & Sclerosis of cord \\
\hline 8 & encephalo- \\
\hline & $p_{n}$ \\
\hline & $\begin{array}{l}\text { Uptic atrophy } \\
\text { Paraplegia }\end{array}$ \\
\hline & Hemiplegia \\
\hline
\end{tabular}

15

\begin{tabular}{l|ll}
- & 0000000000 & 0 \\
- & 0000111000 & 3 \\
- & 0000111100 & 0 \\
- & 0000000000 & 0 \\
0000000000 & 000 \\
- & 0000000000 & 0 \\
- & 0000000000 & 1 \\
0000000000 & 0 \\
- & 0000000000 & 0 \\
- & 0000000000 & 0 \\
- & 0011100000 & 3 \\
- & 0000000000 & 0 \\
- & 0000000000 & 1 \\
0000000000 & 0 \\
- & 0000000000 & 0 \\
- & 0000000000 & 0 \\
- & 0000010000 & 2 \\
0011110000 & 0
\end{tabular}

3200000000

0000000000 0000000000 0000000000

0000000000

1111113111 0000000000

0000000000

0000000000

3000000000

0000000000

1110000000 0000000000

0000000000 0000000000 2200000000

0000000000
000002220000000

000001222220000

000002222220000 000000211000000 090000222200000

000000220000000 000001222000000 000600211000000

000000210000000

000000200000000 000000221000000

000002220000000 000000010000000 000002222000000

000000122200000 000000211100000 000002222220000 000002222100000

Table 6.-Miscellaneous.

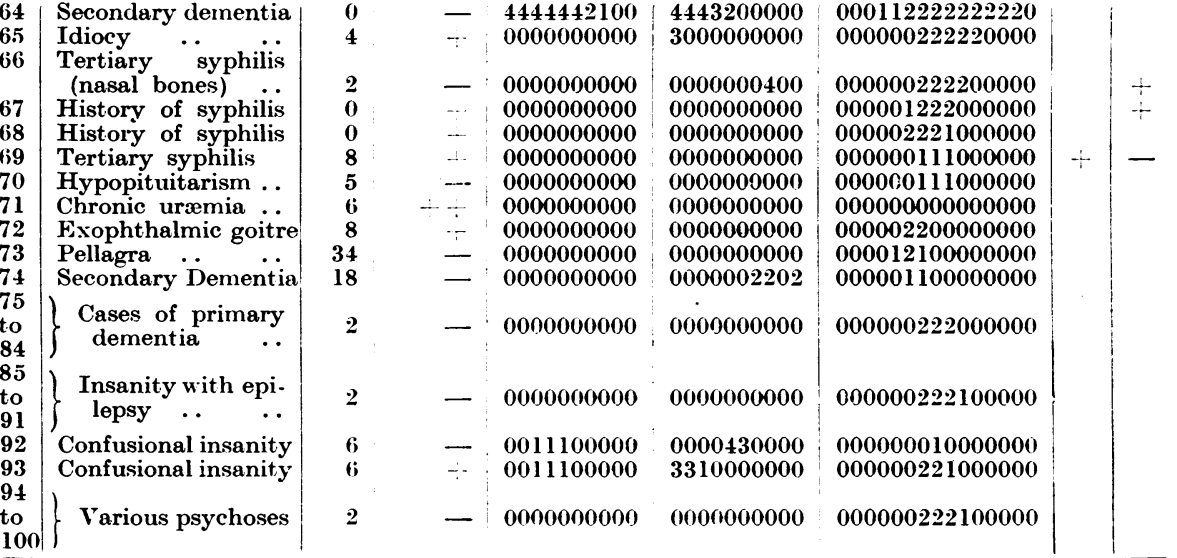


General Discussion.--The types of curve which we obtained when employing the colloidal-gold reaction appeared to follow exactly those which have been described by previous writers. The types of colour change in the various dilutions are certainly of great diagnostic value, but they are not specific for any one condition. A paretic curve was a constant finding in all the spinal fluids taken from general paralytics, but we also obtained four paretic curves in patients who were not suffering from general paralysis. Furthermore, in one advanced case (Case 30) of this disease a negative colloidal-gold reaction was obtained on the first examination of his spinal fluid; when a second examination was made six days later the curve was irregular, and only on the final examination made one month later was there a typical curve. This failure to obtain constant results cannot have been due to faulty solutions of colloidal gold, for the various samples used gave typical curves with other paretic fluids. Other writers have noted this peculiarity, and by some it has been attributed to the provocative effects of lumbar puncture which may determine an altered physico-chemical relation in the globulin of the cerebrospinal fluid.

The gum-mastic reaction has proved in our hands to be less sensitive than Lange's test and of less diagnostic value; but the possibility of technical error seems small, and readings may be rapidly obtained. Against this advantage must be placed the failure to obtain with any degree of constancy curves which could be interpreted as syphilitic.

From a study of 100 spinal fluids, E. Duhot and P. Crampon 5 conclude that the colloidal-benzoin reaction is negative in nonsyphilitic lesions of the nervous system, and positive in 90 per cent of cases of neurosyphilis. In our series the colloidal-benzoin test was positive in every case of general paralysis, and we did not obtain a paretic curve in any case of an obviously non-syphilitic character. In two cases of disseminated sclerosis the type of curve was syphilitic, and with these two exceptions the reaction appeared specific for neurosyphilis. The results were therefore not only in close agreement with those of the colloidal-gold reaction, but in some respects of greater diagnostic value.

When we came to chart the precipitation curves in the benzoin reaction we were at once impressed by the irregular behaviour of many fluids. Indeed, at first glance the test seemed to possess no value, for the presence of varying degrees of precipitation in one or more tubes was an almost constant finding with normal fluids. Thus, in a miscellaneous group of 35 normal spinal fluids the benzoin reaction showed a complete absence of precipitation in the whole 15 tubes in only two instances, a very different finding from the 27 entirely 
negative gold reactions in the same series. However, on carrying our analysis a little further it became evident that this precipitation occurred almost constantly in the middle series, and with one exception was never found in the first five or six tubes. Another peculiarity was the incompleteness of precipitation in tube 1 , even when reduction was pronounced in nearly all the remaining tubes of the series. The method of Guillain, Laroche, and Lechelle seemed, therefore, to stand in need of modification ; but, by restricting the number of dilutions employed to five, we were able to obtain reliable information without altering the strength of the salt solution. Furthermore, the omission of the first tube of the series reduced the quantity of cerebrospinal fluid required for the test by nearly one-half. The benzoin curves in our tables were obtained by employing the original method; but, in the modification proposed, the dilution of cerebrospinal fluid in the first tube is 1 in 2 , and is carried only as far as the fifth tube, the sixth serving as a control.* In the original method of Emanuel ${ }^{7}$ the mastic test was performed with five tubes, and since neither reaction appears to give positive results in cases of meningitis (non-syphilitic) we believe no advantage is obtained by using dilutions higher than $\mathbf{1}$ in $\mathbf{3 2}$.

Guillain and his associates recommend the use of the ultra-microscope for examining the benzoin reaction in its early stages. When the reaction is positive there is an immediate arrest of Brownian movement in the resinous suspension, followed by the appearance

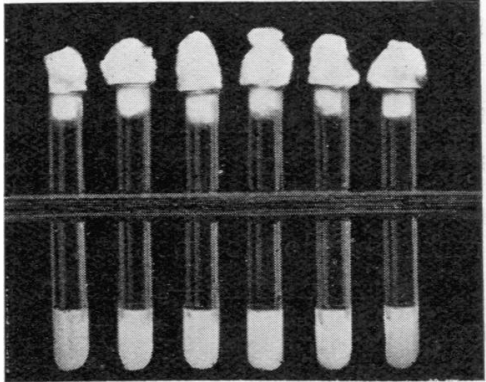

1 in $2 \quad 1$ in $4 \quad 1$ in $8 \quad 1$ in $16 \quad 1$ in 32 Control

Fig. 7.- Colloidal-benzoin reaction (modified). (a) Negative curve.

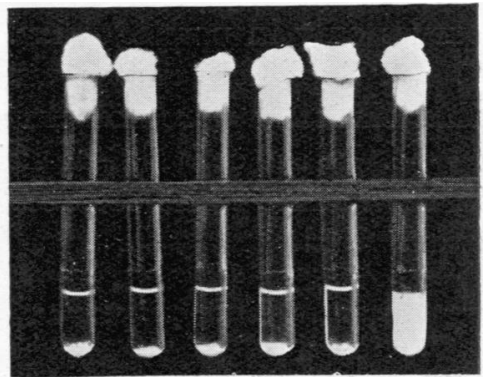

1 in $2 \quad 1$ in $4 \quad 1$ in $8 \quad 1$ in $16 \quad 1$ in 32 Control

FIG. 8.-Colloidal-benzoin reaction (modified). (b) Paretic curve.

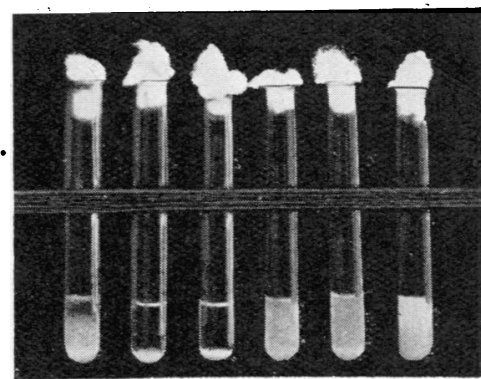

1 in 21 in $4 \quad 1$ in 8 in 16 in 32 Control

FIG. 9.-Colloidal-benzoin reaction (modified). (c) Syphilitic curve. of a mass of refractile granules which agglutinate. We have found

* Our investigation was complete before the appearance of a recent communication (Presse médicale, 1921, Sept. 28), in which Guillain, Laroche, and Lechelle describe a modification of their test, substantially the same as that outlined above. 
that equally reliable information may be obtained by centrifuging the tubes for a few minutes. The control tube in a positive reaction shows no precipitation, and the degrees of reduction in the other tubes are strictly comparable with those obtained by the ordinary technique.

In this test the ease with which the degrees of precipitation may be read, the restriction to three numerals for designating these degrees, and the rapidity with which results can be obtained, may all be claimed as advantages which the colloidal-gold and gum-mastic reactions do not possess.

Conclusions.-1. - In a comparative study of spinal fluids in $\mathbf{3 0}$ cases of general paralysis the gold-sol test and the colloidal-benzoin reaction of Guillain, Laroche, and Lechelle gave parallel results; the gum-mastic reaction was in less close agreement.

2. The colloidal-gold and the gum-mastic tests gave paretic curres in non-paretic fluids. The colloidal-benzoin test gave paretic curves only in paretic fluids.

3. Syphilitic curres were obtained in all three tests from cases that clinically were not examples of neurosyphilis.

4. In the colloidal-benzoin reaction a slight degree of precipitation in the middle series of tubes was often obtained with normal cerebrospinal fluid.

\section{REFERENCES.}

1 Miller, Bresh, Hammers, and Feltox, Johns IIop. Iosp. Bull., 1915, xxvi, 391. 2 Cotring, Jour. Amer. Med. Assoc., 1917, leviii, 1810.

3 Sirth, Med. Recorl, 1917, xcii, 675.

4 Geillar, Laroche, and Lechelde, ('omptes rent. Soc. de Biol., 1920, 1xxxiii, 1077.

¿ Denot and ('ranipon, Bull. Soc. méd. IIôp. de Paris, 1921, xxxvii, 307.

${ }^{6}$ Bribe and Lonkxy, Arch. of Internal Med., 1911, July, 38.

; Exavura, Berl. Klin., 1915, lii, 792. 